\title{
Small Bowel Abnormalities in Patients with Compensated Liver Cirrhosis
}

\author{
Taiki Aoyama $\cdot$ Shiro Oka $\cdot$ Hiroshi Aikata $\cdot$ \\ Makoto Nakano $\cdot$ Ikue Watari $\cdot$ Noriaki Naeshiro • \\ Shigeto Yoshida $\cdot$ Shinji Tanaka $\cdot$ Kazuaki Chayama
}

Received: 30 July 2012/Accepted: 20 November 2012/Published online: 18 December 2012

(C) The Author(s) 2012. This article is published with open access at Springerlink.com

\begin{abstract}
Background Although capsule endoscopy is available as a minimally invasive imaging technique that contributes significantly to the detection of small bowel lesions, there are only a very few published descriptions of small bowel abnormalities in patients with portal hypertension.

Aims The aim of this study was to characterize the occurrence of small bowel lesions by means of capsule endoscopy in patients with portal hypertension, particularly
\end{abstract}

\footnotetext{
T. Aoyama $\cdot$ H. Aikata $\cdot$ M. Nakano $\cdot$ I. Watari $\cdot$ N. Naeshiro $\cdot$

K. Chayama

Department of Gastroenterology and Metabolism, Graduate

School of Biomedical Sciences, Hiroshima University,

1-2-3 Kasumi, Minami-ku, Hiroshima 734-8551, Japan

e-mail: aochin-blue@hiroshima-u.ac.jp

H. Aikata

e-mail: aikata@hiroshima-u.ac.jp

M. Nakano

e-mail: nakano0628@hiroshima-u.ac.jp

I. Watari

e-mail: ikuen@hiroshima-u.ac.jp

N. Naeshiro

e-mail: nn2001aaa@hiroshima-u.ac.jp

K. Chayama

e-mail: chayama@mba.ocn.ne.jp

S. Oka $(\bowtie) \cdot$ S. Yoshida $\cdot$ S. Tanaka

Department of Endoscopy, Hiroshima University Hospital,

1-2-3 Kasumi, Minami-ku, Hiroshima 734-8551, Japan

e-mail: oka4683@hiroshima-u.ac.jp

S. Yoshida

e-mail: yoshida7@hiroshima-u.ac.jp

S. Tanaka

e-mail: colon@hiroshima-u.ac.jp
}

those with compensated liver cirrhosis and associated anemia.

Methods Sixty consecutive patients who met our criteria underwent capsule endoscopy. The frequency, type, and distribution of small bowel lesions were determined, and clinical factors associated with the lesions were examined. Results Small bowel abnormalities were found in 40 patients $(67 \%)$, including erythema $(n=32,53 \%)$, erosion $(n=10,17 \%)$, angioectasia $(n=9,15 \%)$, varices $(n=4,7 \%)$, and villous edema $(n=4,7 \%)$. Most lesions were located in the proximal or middle small bowel. Factors associated with the lesions were ChildPugh class B (vs. class A, $P=0.0023$ ), ascites (vs. no ascites, $P=0.0085$ ), and portal hypertensive gastropathy (vs. no portal hypertensive gastropathy, $P=0.0434$ ).

Conclusions We found capsule endoscopy to be a useful diagnostic modality for detecting clinically significant small bowel lesions in patients with compensated liver cirrhosis. Based on our results, we suggest that this procedure should be especially considered for patients with Child-Pugh class B disease, ascites, and/or portal hypertensive gastropathy if they show evidence of gastrointestinal blood loss and/or iron-deficiency anemia.

Keywords Compensated liver cirrhosis - Portal hypertension - Small bowel lesion - Capsule endoscopy

\section{Introduction}

Portal hypertension (PHT) is a frequent manifestation of liver cirrhosis (LC), and it can cause various pathological changes in the entire gastrointestinal (GI) tract (from the esophagus to the anus) [1-3]. Associated changes in the stomach and colon are well described in the literature, 
usually on the basis of upper GI endoscopy and colonoscopy, respectively. It is very likely that PHT induces changes in the mucosa of the small bowel in patients with LC [2, 4-7]. De Palma et al. [4] defined these changes as portal hypertensive enteropathy (PHE), which presents as inflammatory-like lesions (edematous, erythematous, granular, and friable lesions) and/or vascular lesions (cherry red spots, telangiectasias, or angiodysplasia-like lesions and varices). However, the clinical background and clinical implications of such changes are largely unknown.

According to De Palma et al. [4], PHE is significantly associated with Child-Pugh class $\mathrm{C}$ cirrhosis. These authors also recommend that patients with Child-Pugh class $\mathrm{C}$ cirrhosis, large esophageal varices, portal hypertensive gastropathy (PHG), and/or portal hypertensive colopathy (PHC) be examined by capsule endoscopy (CE) as a matter of routine. PHE may have clinical significance as a potential source of overt or occult GI bleeding in 5.5-11\% of patients with PHT [4, 6]. An episode of such bleeding in patients with advanced liver disease can decompensate the patient and may be fatal. The GI tract, including the small bowel, should be examined early in the course of the diagnostic workup. However, whether $\mathrm{CE}$ should be undertaken in patients with compensated LC remains unclear. The prevalence of associated small bowel lesions and the clinical factors related to such lesions are also unknown.

We conducted a CE-based study to evaluate (1) the prevalence, types, and anatomic distribution of small bowel lesions associated with PHT in patients with compensated LC; (2) the association of these lesions with clinical factors; (3) whether these lesions can be the cause of obscure bleeding. Finally, we clarified the clinical significance of examining the small bowel in patients with compensated LC.

\section{Methods}

\section{Patients}

The study cohort comprised 60 consecutive patients with compensated LC and associated anemia (35 men and 25 women; mean age 68.8 years; age range $27-87$ years) who underwent $\mathrm{CE}$ at Hiroshima University Hospital between March 2010 and February 2012. The hemoglobin level in these patients was $\leq 12.0 \mathrm{~g} / \mathrm{dL}$, but neither sites of active bleeding nor evidence of bleeding were identified upon upper GI endoscopy and colonoscopy. Eight (13\%) patients had a history of GI bleeding.

The liver disease was of viral etiology in 47 patients [due to hepatitis $\mathrm{C}$ virus (HCV) in 39 patients and $\mathrm{HBV}$ in 8], of non-viral etiology in ten patients (excessive alcohol consumption in 5 patients, non-alcoholic steatohepatitis in 4 , and primary biliary cirrhosis in 1), and of unknown etiology in three patients. LC was diagnosed in each case on the basis of the patient's life and medical histories, indicative physical findings (e.g., vascular spiders, palmar erythema, leg edema), laboratory findings (impaired synthetic liver function and thrombocytopenia), radiographic features of the upper abdomen (nodular appearance, irregular liver contour, splenomegaly, and/or ascites), and/or liver biopsy findings (F4 fibrosis, according to the New Inuyama Classification system). LC was graded for severity according to the ChildPugh classification system. Only patients with Child-Pugh class $\mathrm{A}(n=33)$ or $\mathrm{B}(n=27)$ disease were included in the study; those with Child-Pugh class $\mathrm{C}$ disease (decompensated LC) were excluded. For this study, compensated liver cirrhosis was defined as Child-Pugh class A or B cirrhosis, i.e., cirrhosis limited to hepatic impairment without progressive jaundice, refractory ascites, or severe hepatic encephalopathy. No patient using non-steroidal antiinflammatory drugs or $\beta$-blockers was included.

Written informed consent was obtained from all patients who participated in the study. The study protocol conformed to the guidelines of the 1975 Declaration of Helsinki (sixth revision, 2008) as reflected in a priori approval by the institutional review board of Hiroshima University Hospital.

\section{Endoscopic Examinations}

All patients underwent computed tomography (CT) to rule out bowel obstruction and to confirm the presence or absence of HCC, portal vein tumor thrombus, and ascites. Transabdominal ultrasonography was performed if necessary. Upper GI endoscopy and colonoscopy were performed independently, and the presence or absence of esophageal varices, gastric varices, $\mathrm{PHG}$, and PHC was determined.

$\mathrm{CE}$ was later performed with a video capsule (PillCam SB2; Given Imaging Ltd, Yokneam, Israel). The capsule was swallowed with a solution of dimethicone after an overnight fast, without any other preparation. Two and four hours after swallowing the capsule, patients were allowed to drink clear liquids and to eat a light meal, respectively; after $8 \mathrm{~h}$, the sensor array and recording device were removed. Images were analyzed with Rapid Reader 6 software on a RAPID 5 or 6.5 workstation (software and workstation from Given Imaging Ltd). Three experienced endoscopists were present at every reading so that diagnoses were reached by agreement. Interpreters were blinded to the patients' clinical and endoscopic information.

\section{Study Measures}

The number, type, and location of small bowel lesions found upon CE were determined in each patient. Diagnosis of PHE was in accordance with the criteria described by De Palma et al. [4]. The entire small bowel was divided into 
three parts based on transit time: the proximal, middle, and distal small bowel. Clinical factors were examined in relation to the lesions, i.e., sex, age, liver function (ChildPugh score), etiology of the cirrhosis (viral/nonviral), laboratory values (hemoglobin, serum ferritin, serum iron, total bilirubin, aspartate transaminase, alanine transaminase, albumin, prothrombin time, platelet count), and presence or absence of esophageal varices, gastric varices, PHG, PHC, hepatocellular carcinoma (HCC), portal vein tumor thrombus, and ascites.

\section{Statistical Analysis}

Values are shown as the mean \pm standard deviation (SD). Data were analyzed by Student's $t$ test, the chi-square test with Yates correction, or the Fisher's exact test, as appropriate. All tests were two-tailed, and $P<0.05$ was considered statistically significant. All statistical calculations were done with Microsoft Excel 2008 for Mac (Microsoft, Redmond, WA).

\section{Results}

\section{Endoscopic Findings}

There was no capsule retention, and exit of the capsule was confirmed in all patients.
Total capsule enteroscopy was achieved in 55 patients (92\%). Imaging in the remaining five patients was nearly complete, as confirmed by the identification of lymphoid follicles in the terminal ileum.

Abnormalities in the small bowel, i.e., PHE, were found by CE in 40 patients $(67 \%)$ and presented as erythema ( $n=32$ patients, $53 \%$ ), erosion $(n=10$ patients, $17 \%)$, angioectasia $(n=9$ patients, $15 \%)$, varices $(n=4$ patients, $7 \%)$, and villous edema $(n=4$ patients, $7 \%)$. The endoscopic appearances of these abnormalities are shown in Fig. 1. Most lesions were found to be located in the proximal or middle small bowel (Table 1). Villous edema was also located mainly in the proximal and/or middle small bowel.

Upper GI endoscopy and colonoscopy revealed the following esophageal varices: gastric varices, $\mathrm{PHG}$, and PHC in 34 (57\%), 11 (18\%), 13 (22\%), and 12 patients (20\%), respectively; CT revealed HCC, portal vein tumor thrombus, and ascites in $42(70 \%)$, seven (12\%), and 20 patients $(33 \%)$, respectively (Table 2 ).

\section{Relations Between Clinical Factors and Small Bowel Lesions}

To investigate the relations between small bowel lesions and clinical factors, we compared patients with and without small bowel lesions, as shown in Table 2. Small bowel
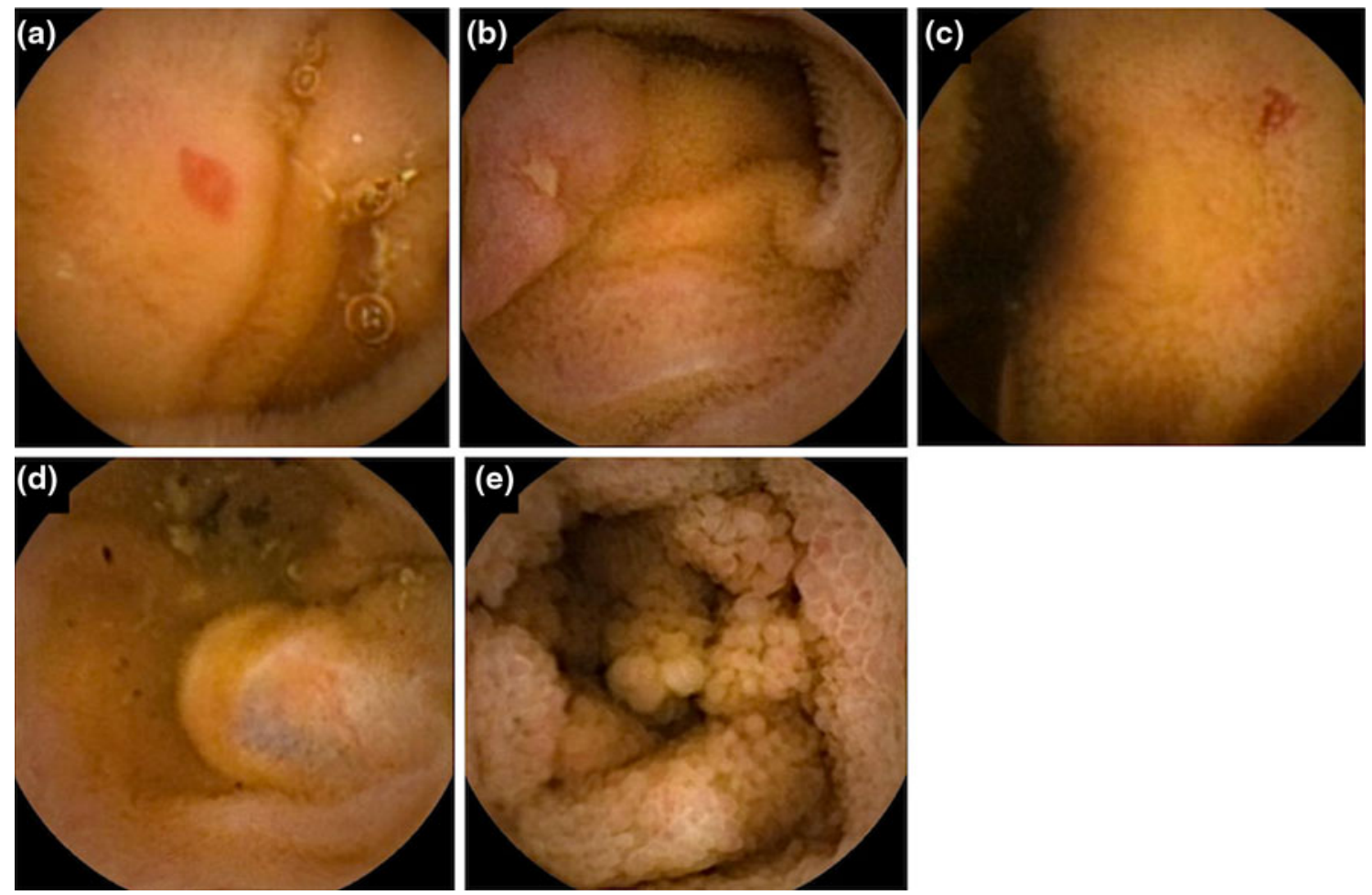

Fig. 1 Endoscopic appearance of small bowel lesions related to portal hypertension in patients with liver cirrhosis. a Erythema, b erosion, c angioectasia, d varices, e villous edema 
Table 1 Number and distribution of lesions other than villous edema in the small bowel

\begin{tabular}{llll}
\hline Lesion type & \multicolumn{2}{l}{ Small bowel segment ${ }^{\mathrm{a}}$} \\
\cline { 2 - 4 } & Proximal third & Middle third & Distal third \\
\hline Erythema & 35 & 20 & 15 \\
Erosion & 10 & 8 & 4 \\
Angioectasia & 8 & 2 & 2 \\
Varices & 1 & 4 & 1 \\
Total & $54(49 \%)$ & $34(31 \%)$ & $22(20 \%)$ \\
\hline
\end{tabular}

a The entire small bowel was divided into three parts, and the location of lesions was determined according to capsule transit time

lesions were more prevalent among patients with ChildPugh class B disease than among those with Child-Pugh class A disease $(P=0.0023)$. Ascites and PHG were significantly more prevalent in patients with small bowel lesions than in those without such lesions $(P=0.0085$ and 0.0434 , respectively). Age, sex, etiology, esophageal varices, gastric varices, $\mathrm{PHC}, \mathrm{HCC}$, and portal vein tumor thrombus did not differ between patients with and without small bowel lesions. Laboratory values, including hemoglobin $(9.6 \pm 1.6$ vs. $9.7 \pm 2.3 \mathrm{~g} / \mathrm{dL})$, serum iron $(71 \pm$ 58 vs. $46 \pm 26 \mu \mathrm{g} / \mathrm{mL})$, and serum ferritin $(167 \pm 107$ vs. $60.8 \pm 126 \mathrm{ng} / \mathrm{dL}$ ), which were of particular interest, did not differ statistically between patients with and without small bowel lesions (Table 3). In multivariate analysis, ascites (odds ratio 5.67, $95 \%$ confidence interval 1.31-39.6; $P=0.037)$ was shown to be a significant predictor of PHE.

Although none of the study patients were experiencing active GI bleeding at the time of our investigation, eight patients $(13 \%)$ had a history of such bleeding, as noted above. Angioectasia was found in two of these eight patients and considered to be the source of previous bleeding; the sources were not evident in the other six patients.

We used CE to explore the small bowel in all patients, and we added double-balloon endoscopy (DBE) when indicated. DBE was used for large CE-identified angioectasias ( $n=4$ patients), for further examination and/or treatment of CE-identified varices ( $n=2$ patients), and to rule out varices when $\mathrm{CE}$ indicated a submucosal tumorlike elevation ( $n=5$ patients). Among our patients, DBE did not detect any abnormalities that had not been detected by $\mathrm{CE}$. We confirmed angioectasia in the jejunum in two patients and in the ileum in two patients, all of which were successfully treated by polidocanol injection therapy. Hemoglobin levels improved in all patients who received treatment with the DBE procedure. Moreover, we also confirmed varices in two patients, both of whom were simply monitored because the varices were deemed unlikely to bleed.
Table 2 Clinical characteristics of total patients and of patients per group (patients with and without small bowel lesions)

\begin{tabular}{|c|c|c|c|c|}
\hline \multirow[t]{2}{*}{ Characteristic } & \multirow{2}{*}{$\begin{array}{l}\text { Total } \\
(n=60)\end{array}$} & \multicolumn{2}{|l|}{ Group } & \multirow[t]{2}{*}{$P$ value $^{\mathrm{a}}$} \\
\hline & & $\begin{array}{l}\text { Lesions } \\
\text { present } \\
(n=40)\end{array}$ & $\begin{array}{l}\text { Lesions } \\
\text { absent } \\
(n=20)\end{array}$ & \\
\hline $\begin{array}{l}\text { Age (years, } \\
\text { mean } \pm \mathrm{SD})\end{array}$ & $68.8 \pm 11.8$ & $69.8 \pm 12.6$ & $66.8 \pm 9.9$ & 0.3592 \\
\hline Sex ratio (male/female) & $35 / 25$ & $24 / 16$ & $11 / 9$ & 0.9262 \\
\hline \multicolumn{5}{|l|}{ Child-Pugh class } \\
\hline A & $35(55)$ & $17(43)$ & $16(80)$ & \multirow[t]{2}{*}{$0.0023^{\mathrm{c}}$} \\
\hline $\mathrm{B}$ & $27(45)$ & $23(57)$ & $4(20)$ & \\
\hline \multicolumn{5}{|l|}{ Etiology of LC } \\
\hline Viral & & & & \multirow[t]{8}{*}{$0.1860^{\mathrm{d}}$} \\
\hline $\mathrm{HCV}$ & $39(65)$ & $26(65)$ & $13(65)$ & \\
\hline $\mathrm{HBV}$ & $8(13)$ & $3(7)$ & $5(25)$ & \\
\hline \multicolumn{4}{|l|}{ Nonviral } & \\
\hline Alcohol & $5(8)$ & $4(10)$ & $1(5)$ & \\
\hline NASH & $4(7)$ & $3(7)$ & $1(5)$ & \\
\hline PBC & $1(2)$ & $1(4)$ & $0(0)$ & \\
\hline Unknown & $3(5)$ & $3(7)$ & $0(0)$ & \\
\hline \multicolumn{5}{|l|}{ Endoscopic findings ${ }^{\mathrm{b}}$} \\
\hline E. varices & $34(57)$ & $25(63)$ & $9(45)$ & 0.3110 \\
\hline G. varices & $11(18)$ & $9(23)$ & $2(10)$ & 0.3024 \\
\hline PHG & $13(22)$ & $12(30)$ & $1(5)$ & 0.0434 \\
\hline PHC & $12(20)$ & $10(25)$ & $2(10)$ & 0.1920 \\
\hline \multicolumn{5}{|l|}{ CT findings } \\
\hline HCC & $42(70)$ & $30(75)$ & $12(60)$ & 0.3700 \\
\hline $\begin{array}{l}\text { Portal vein } \\
\text { tumor thrombus }\end{array}$ & $7(12)$ & $5(13)$ & $2(10)$ & 1.0000 \\
\hline Ascites & $20(33)$ & $18(45)$ & $2(10)$ & 0.0085 \\
\hline
\end{tabular}

Data are presented as the number of patients with the percentage given in parenthesis, unless otherwise indicated

$L C$ Liver cirrhosis, $N A S H$ non-alcoholic steatohepatitis, $P B C$ primary biliary cirrhosis, E. varices esophageal varices, $G$. varices gastric varices, $P H G$ portal hypertensive gastropathy, $P H C$ portal hypertensive colopathy, $H C C$ hepatocellular carcinoma, $S D$ standard deviation

${ }^{a}$ Comparisons were performed using the chi-square test except for the variable "age" for which Student's $t$ test was used

${ }^{b}$ By upper gastrointestinal endoscopy or colonoscopy

${ }^{c}$ Child-Pugh class A vs. class B

${ }^{\mathrm{d}}$ Viral vs. nonviral/unknown

\section{Discussion}

Portal hypertension is one of the main complications of LC. It results from a combination of increased intrahepatic vascular resistance and increased blood flow through the portal venous system. These vascular changes are mediated, in part, by imbalances in the vasoconstrictors and vasodilators in both the liver and the splanchnic circulation. Although the liver is the primary organ damaged in cases of LC, pathological lesions are not limited to the liver. The altered circulatory hemodynamics contributes to 
Table 3 Laboratory values in total patients and per group (patients with and without small bowel lesions)

\begin{tabular}{lccc}
\hline Laboratory value & Total $(n=60)$ & Group & $P$ value \\
\cline { 3 - 4 } & & $\begin{array}{l}\text { Lesions present } \\
(n=40)\end{array}$ & $\begin{array}{l}\text { Lesions absent } \\
(n=20)\end{array}$ \\
\hline Hemoglobin $(\mathrm{g} / \mathrm{dL})$ & $9.7 \pm 1.9$ & $9.6 \pm 1.6$ & $9.7 \pm 2.3$ \\
Serum iron $(\mu \mathrm{g} / \mathrm{dL})$ & $63 \pm 51$ & $71 \pm 58$ & $46 \pm 26$ \\
Serum ferritin (ng/dL) & $92.0 \pm 156$ & $167 \pm 107$ & $60.8 \pm 126$ \\
T.Bil (mg/dL) & $1.0 \pm 0.5$ & $1.0 \pm 0.6$ & $0.9 \pm 0.3$ \\
AST (IU/L) & $41 \pm 18$ & $39 \pm 17$ & $44 \pm 20$ \\
ALT (IU/L) & $33 \pm 19$ & $32 \pm 20$ & $35 \pm 17$ \\
Alb $(\mathrm{g} / \mathrm{dL})$ & $3.5 \pm 0.5$ & $3.4 \pm 0.5$ & $3.6 \pm 0.5$ \\
PT $(\%)$ & $75 \pm 15$ & $74 \pm 16$ & $78 \pm 14$ \\
Platelets $\left(\times 10^{4} \mu \mathrm{L}\right)$ & $11 \pm 6.2$ & $10 \pm 6.1$ & $12 \pm 6.3$ \\
\hline
\end{tabular}

T.Bil Total bilirubin, $A S T$ aspartate transaminase, $A L T$ alanine transaminase, $A l b$ albumin, $P T$ prothrombin time

Values are shown as the mean $\pm \mathrm{SD}$

Comparisons were performed with the Student's $t$ test

the syndrome, resulting in the spectrum of gastropathy, colopathy, and enteropathy. According to Jonas et al. [8] PHT alters the digestive tract mucosa and increases susceptibility to injury. Impaired oxygenation of the mucosa, and hence ischemia, is the probable mechanism for this increased susceptibility [9]; decreased hemoglobin oxygen saturation has been documented in the duodenal mucosa of patients with LC [10]. The GI tract of patients with LC is subject to various pathological lesions. Mucosal edema, erythema, angioectasia, and varices have been detected mainly in the stomach and colon of such patients. However, it is very likely that PHT induces the same changes in the small bowel of patients with LC $[4,5]$. With the development of $\mathrm{CE}$, such changes are no longer a matter of speculation. CE and DBE are nearly equal in their ability to detect small bowel lesions [11, 12], and we found that no CE- or DBE-related complications occurred in our patients. $\mathrm{CE}$ is now widely accepted as the gold standard noninvasive technique for evaluating obscure GI bleeding. It is possible to obtain clear CE images with the proper preparation [13]. Moreover, new technologies, such as CE with flexible imaging color enhancement, have improved the visibility and detectability of small bowel lesions $[14,15]$. Therefore, CE has become a superior diagnostic modality for screening patients with LC [16].

De Palma et al. [4] reported PHE in $67.5 \%$ of patients with PHT. In a clinical study described by Akyuz et al. [17] small bowel abnormalities were found in $90.5 \%$ of patients with PHT. In our study, small bowel abnormalities were detected by CE in $67 \%$ of our patients. Higaki et al. [18] noted that villous edema, with its characteristic herring roe appearance, may be a feature of advanced LC. We actually observed villous edema in four $(7 \%)$ of our patients, all of which had Child-Pugh class B disease with ascites. Most of the lesions, including villous edema, were observed in the proximal and middle small bowel. Nagral et al. [19] reported congestive jejunopathy in $84 \%$ of patients with PHT. Further, increased superior mesenteric and splanchnic artery blood flow has been reported in patients with PHT, pointing to the possibility of congestion in the superior mesenteric circulation [20]. Decreased blood flow velocity and increased thickness of the superior mesenteric vein are associated with small bowel injury [21].

In the early stages of LC, patients have a normal portal pressure gradient $(3-6 \mathrm{mmHg}$ ); however, as the disease progresses, portal pressure increases to abnormal levels. The hepatic venous pressure gradient (HVPG), which accurately reflects portal pressure [22, 23], correlates with the Child-Pugh status, being higher in patients with ChildPugh class B cirrhosis than in those with Child-Pugh class A cirrhosis [24, 25]. The severity of PHT correlates with the severity of liver disease. In the study by De Palma et al. [4], the prevalence of PHE was shown to increase with worsening Child-Pugh class. Moreover, HVPG was shown to be higher in patients with ascites than in those without ascites [24, 25]. We detected a greater number of small bowel lesions among Child-Pugh class B patients than among Child-Pugh class A patients. We also detected a greater number of small bowel lesions among patients with ascites than among those without ascites. The relation between the existence of small bowel lesions and the presence of ascites has not been explored in detail. The mechanism by which ascites develops in patients with cirrhosis is multifactorial, with the largest contribution from severe PTH. Takahashi et al. [26] showed that villous edema correlated most strongly with HVPG of the small bowel lesions. Therefore, both ascites and small bowel 
lesions appear to develop on the basis of PHT. Ascites was shown in our analysis to be a significant predictor of PHE. Kim et al. [27] showed HVPG to be significantly higher in patients with PHG than in those without PHG. One group of investigators found PHE to be significantly prevalent in the presence of PHG and/or PHC [4], although Repici et al. [28] found no association between PHE, as documented by $\mathrm{CE}$, and the presence of PHG and/or PHC. Nagral et al. [19] correlated the presence of congestive jejunopathy with that of histological congestive gastropathy in the fundus, which suggests that small bowel lesions, especially in the proximal small bowel, are related to the presence of gastric congestion, i.e., PHG. Our study revealed a relation between PHG and PHE. Also, HVPG is reported to be significantly lower in the presence of gastric varices than in their absence [29]. We found no increase in the prevalence of small bowel lesions in patients with gastric varices.

Chronic anemia is frequently observed in patients with LC. GI bleeding is a common complication of PHT, and acute or chronic blood loss may contribute to the development of anemia. On the basis of some reports, we can assume that PHG and PHE lesions are implicated in GI bleeding $[30,31]$. Portal hypertensive intestinal vasculopathy most often involves the stomach and is a common source of bleeding. The annual incidence of minor mucosal blood loss without overt bleeding is increased to about $8 \%$ in patients with mild PHG and to up to $25 \%$ in patients with severe PHG [32]. Ganguly et al. [33] observed overt bleeding in 8 and $4 \%$ of patients with rectal varices and colopathy, respectively. PHE may also have clinical significance as a potential source of overt or occult GI bleeding [4, 6]. Moreover, small bowel angioectasias and varices are thought to account for unexplained blood loss in portal hypertensive patients [17, 30]. Yamada et al. [34] specifically reported angiodysplasia as the main cause of acute bleeding from the small bowel in patients with PTH. It is estimated that only $10 \%$ of all patients with angioectasia have clinically evident GI bleeding during their lifetime [35] but that these lesions account for up to $80 \%$ of all cases of obscure bleeding in patients with ongoing transfusion requirements [35]. After the bleeding has stopped, it is difficult to identify angioectasias as the sources of bleeding in patients with PHE if they are tiny [35]. Thus, Kodama et al. [36] recommend that the small bowel be examined as soon as possible because angioectasia can cause anemia. Although there was not a significant difference in our study, the hemoglobin level was lower in patients with small angioectasias than in those without such lesions ( 8.9 vs. $9.7 \mathrm{~g} / \mathrm{dL} ; P=0.22$ ). Variceal lesions may also be the source of bleeding; however, they are sometimes difficult to differentiate from submucosal tumors [37]. Further examination should be considered as appropriate in some cases. Therefore, angioectasias or suspected variceal lesions which are likely to bleed should be further examined as appropriate. In this study, we failed to prove that PHE contributed largely to the development of anemia: there was no significant difference in hemoglobin levels between patients with PHE and those without PHE $(9.6 \pm 1.6$ vs. $9.7 \pm 2.3 \mathrm{~g} / \mathrm{dL} ; P=0.87)$. We suggest that this lack of a significant different is because the mechanism by which anemia develops in patients with cirrhosis is multifactorial. However, based on the results of other published studies [4, 6, 17, 18, 30, 31, 34-36], we are confident that PHE lesions can be the cause of bleeding and subsequent anemia.

We conclude that $\mathrm{CE}$ is a useful diagnostic modality for detecting clinically significant small bowel lesions in patients with compensated LC and associated anemia. Although the clinical relevance of our findings needs to be further explored, $\mathrm{CE}$ should be considered for patients with compensated cirrhosis that falls into Child-Pugh class B, is characterized by ascites, and/or is characterized by PHG-if there is evidence of GI blood lost and/or iron deficiency anemia.

Acknowledgments The study was supported by departmental resources only.

\section{Conflict of interest None.}

Open Access This article is distributed under the terms of the Creative Commons Attribution Noncommercial License which permits any noncommercial use, distribution, and reproduction in any medium, provided the original author(s) and the source are credited.

\section{References}

1. Sarfeh IJ, Tarnawaski A. Gastric mucosal vasculopathy in portal hypertension. Gastroenterology. 1987;93:1129-1131.

2. Viggiano TR, Gostout CJ. Portal hypertensive intestinal vasculopathy: a review of the clinical, endoscopic, and histopathologic features. Am J Gastroenterol. 1992;87:944-954.

3. Kozarek RA, Botoman VA, Bredfeldt JE, Roach JM, Patterson DJ, Ball TJ. Portal colonopathy: prospective study of colonoscopy in patients with portal hypertension. Gastroenterology. 1991;101:1192-1197.

4. De Palma GD, Rega M, Masone S, et al. Mucosal abnormalities of the small bowel in patients with cirrhosis and portal hypertension: a capsule endoscopy study. Gastrointest Endosc. 2005;62: 529534.

5. Misra SP, Dwivedi M, Misra V, Gupta M. Ilial varices and portal hypertensive ileopathy in patients with cirrhosis and portal hypertension. Gastrointest Endosc. 2004;60:778-783.

6. Figueiredo P, Almeida N, Lérias C, et al. Effect of portal hypertension in the small bowel: an endoscopic approach. Dig Dis Sci. 2008;53:2144-2150.

7. Kovács M, Pák P, Pák G, Fehér J, Rácz I. Small bowel alterations in portal hypertension: a capsule endoscopic study. Hepatogastroenterology. 2009;56:1069-1073.

8. Jonas G, Erickson RA, Morgan T. Effect of portal hypertension on in vivo bile acid mediated small intestinal mucosal injury in the rat. Dig Dis Sci. 1990;35:743-748. 
9. Sarfeh IJ, Soliman H, Waxman K, et al. Impaired oxygenation of gastric mucosa in portal hypertension. The basis for increased susceptibility to injury. Dig Dis Sci. 1989;34:225-228.

10. Tanaka $M$, Inatsuchi $S$, Terasaki $\mathrm{T}$, et al. Duodenal mucosal hemodynamics in patients with liver cirrhosis. Acta Med Okayama. 1990;44:273-277.

11. Fukumoto A, Tanaka S, Shishido T, Takemura Y, Oka S, Chayama K. Comparison of detectability of small-bowel lesions between capsule endoscopy and double-balloon endoscopy for patients with suspected small-bowel disease. Gastrointest Endosc. 2009;69:857-865.

12. Shishido T, Oka S, Tanaka S, et al. Diagnostic yield of capsule endoscopy vs. double-balloon endoscopy for patients who have undergone total enteroscopy with obscure gastrointestinal bleeding. Hepatogastroenterology. 2012;59:955-959.

13. Park SC, Keum B, Seo YS, et al. Effect of bowel preparation with polyethylene glycol on quality of capsule endoscopy. Dig Dis Sci. 2011;56:1769-1775.

14. Imagawa $H, O$ Oka $S$, Tanaka $S$, et al. Improved visibility of lesions of the small intestine via capsule endoscopy with computed virtual chromoendoscopy. Gastrointest Endosc. 2011;73:299-306.

15. Imagawa $\mathrm{H}$, Oka $\mathrm{S}$, Tanaka $\mathrm{S}$, et al. Improved detectability of small-bowel lesions via capsule endoscopy with computed virtual chromoendoscopy: a pilot study. Scand J Gastroenterol. 2010; 46:1133-1137.

16. de Franchis R, Dell'Era A, Primignani M. Diagnosis and monitoring of portal hypertension. Dig Liver Dis. 2008;40:312-317.

17. Akyuz F, Pinarbasi B, Ermis F, et al. Is portal hypertensive enteropathy an important additional cause of blood loss in portal hypertensive patients? Scand J Gastroenterol. 2010;45:1497-1502.

18. Higaki $\mathrm{N}$, Matsui $\mathrm{H}$, Imaoka $\mathrm{H}$, et al. Characteristic endoscopic features of portal hypertensive enteropathy. J Gastroenterol. 2008;43:327-331.

19. Nagral AS, Joshi AS, Bhatia SJ, Abraham P, Mistry FP, Vora IM. Congestive jejunopathy in portal hypertension. Gut. 1993;34: 694-697.

20. Zwiebel WJ, Mountford RA, Halliwell MJ, Wells PN. Splanchnic blood flow in patients with cirrhosis and portal hypertension: investigation with duplex Doppler US. Radiology. 1995;194:807-812.

21. Hidenori T, Makoto N, Yozo O, et al. Congestion of superior mesenteric veins and small bowel mucosal injury after endoscopic treatment of esophageal varices in patients with portal hypertension. Dig Dis Sci. 2001;46:2353-2359.

22. Bellis L, Castellacci R, Montagnese F, Festuccia F, Corvisieri P, Puoti C. Hepatic venous pressure gradient determination in patients with hepatitis $\mathrm{C}$ virus-related and alcoholic cirrhosis. Eur J Gastroenterol Hepatol. 2003;15:1085-1089.

23. Perello A, Escorsell A, Bru C, et al. Wedged hepatic venous pressure adequately reflects portal pressure in hepatitis $\mathrm{C}$ virusrelated cirrhosis. Hepatology. 1999;30:1393-1397.
24. Silkauskaite V, Pranculis A, Mitraite D, Jonaitis L, Petrenkiene V, Kupcinskas L. Hepatic venous pressure gradient measurement in patients with liver cirrhosis: a correlation with disease severity and variceal bleeding. Medicina (Kaunas). 2009;45:8-13.

25. Wadhawan M, Dubey S, Sharma BC, Sarin SK. Hepatic venous pressure gradient in cirrhosis: correlation with the size of varices, bleeding, ascites, and Child's status. Dig Dis Sci. 2006;51: 2264-2269.

26. Takahashi Y, Fujimori S, Narahara Y, et al. Small intestinal edema had the strongest correlation with portal venous pressure amongst capsule endoscopy findings. Digestion. 2012;86:48-54.

27. Kim MY, Choi H, Soon Koo Baik SK, et al. Portal hypertensive gastropathy: correlation with portal hypertension and prognosis in cirrhosis. Dig Dis Sci. 2010;55:3561-3567.

28. Repici A, Pennazio M, Ottobrelli A, et al. Endoscopic capsule in cirrhotic patients with portal hypertension: spectrum and prevalence of small bowel lesions (abstract). Endoscopy. 2005;37:A72.

29. Kim MY, Baik SK, Suk KT, et al. Measurement of hepatic venous pressure gradient in liver cirrhosis: relationship with the status of cirrhosis, varices, and ascites in Korea. Korean J Hepatol. 2008;14:150-158.

30. Tang SJ, Zanati S, Dubcenco E, et al. Diagnosis of small-bowel varices by capsule endoscopy. Gastrointest Endosc. 2004;60:129-135.

31. Thiruvengadam R, Gostout CJ. Congestive gastroenteropathyan extension of nonvariceal upper gastrointestinal bleeding in portal hypertension. Gastrointest Endosc. 1989;35:504-507.

32. D'Amico G, Pagliaro L, Bosch J. The treatment of portal hypertension: a metanalytic review. Hepatology. 1995;22:232-254.

33. Ganguly S, Sarin SK, Bhatia V, Lahoti D. The prevalence and spectrum of colonic lesions in patients with cirrhotic and noncirrhotic portal hypertension. Hepatology. 1995;21:1226-1231.

34. Yamada A, Watabe H, Obi S, et al. Surveillance of small intestinal abnormalities in patients with hepatocellular carcinoma: a prospective capsule endoscopy study. Dig Endosc. 2011;23: 124-129.

35. Raju GS, Gerson L, Das A. American Gastroenterological Association. American gastroenterological association (AGA) Institute technical review on obscure gastrointestinal bleeding. Gastroenterology. 2007;133:1697-1717.

36. Kodama M, Uto H, Numata M, et al. Endoscopic characterization of the small bowel in patients with portal hypertension evaluated by double balloon endoscopy. J Gastroenterol. 2008;43:589-596.

37. Watanabe N, Toyonaga A, Kojima S, et al. Current status of ectopic varices in Japan: results of a survey by the Japan Society for Portal Hypertension. Hepatol Res. 2010;40:763-776. 